\title{
NEAR-FIELD EXPANSION OF THE METRIC DUE TO A COSMIC STRING
}

\author{
MALCOLM ANDERSON ${ }^{1}$
}

(Received 16 January 1995; revised 13 March 1996)

\begin{abstract}
By erecting a co-ordinate system tailored to the geometry of a cosmic string and examining the properties of the near gravitational field, it is possible to distinguish two types of gravitational waves supported by a general string metric. The first type, travelling waves, are completely decoupled from the curvature of the world sheet, whereas the second type, which I choose to call curvature waves, are generated in response to any non-trivial geometric structure on the string.
\end{abstract}

\section{Introduction}

Cosmic strings are line vortices of Higgs field energy that may have played an important role in the formation of structure in the early Universe (see [28] for a review of the origins and early history of cosmic-string theory). Whether or not they are ever shown to be an indispensable ingredient of cosmogonic theories, cosmic strings have already provided many valuable insights into classical field theory, Einstein gravitation and the nature of closed timelike curves. However, much more work needs to be done before we can claim an understanding of cosmic strings that is even remotely comparable to our current understanding of black holes, which are in many ways just lower-dimensional analogues of strings.

In this paper, I draw attention to the fact that cosmic strings naturally support two distinct types of gravitational waves: the "travelling waves" previously identified by Vachaspati [26] and Garfinkle [7], and a second type that I will refer to as "curvature waves". Travelling waves arise by superposing plane-fronted gravitational waves on the near-field metric of a cosmic string, whereas curvature waves have a more general structure and (unlike travelling waves) couple directly to the intrinsic curvature of

\footnotetext{
${ }^{1}$ School of Engineering and Mathematics, Edith Cowan University, Joondalup Drive, Joondalup, Western Australia 6027, Australia

(C) Australian Mathematical Society 1999, Serial-fee code 0334-2700/99
} 
the string's world sheet. In establishing this dichotomy, I will outline a method for constructing co-ordinates in a neighbourhood of the world sheet that are tailored to the geometry of the world sheet in a natural way. This co-ordinate system allows us, among other things, to standardise and compare the near-field metrics of the plethora of cosmic-string solutions that have been published over the last thirteen years.

The metric outside an infinite straight string was discovered, independently, by Gott and Hiscock $[10,12]$ in 1985 - although it should be noted that the weak-field limit (which scarcely differs from the full solution) was published four years earlier by Vilenkin [27]. It is arguably the simplest interesting solution of Einstein's field equations. In cylindrical co-ordinates $(t, r, z, \phi)$, the line element reads:

$$
d s^{2}=d t^{2}-d z^{2}-d r^{2}-(1-4 \mu)^{2} r^{2} d \phi^{2}
$$

where $\mu$ is a constant lying in the range $[0,1 / 4)$. Geometrically, the line element describes Minkowski spacetime with a wedge of angular extent $\Delta \theta=8 \pi \mu$ removed from along the $z$-axis.

By defining $x=(\alpha r)^{1 / \alpha} \cos \phi$ and $y=(\alpha r)^{1 / \alpha} \sin \phi$, where $\alpha=1-4 \mu$, it is possible to rewrite the line element (1) in what are often called "isotropic" coordinates:

$$
d s^{2}=d t^{2}-d z^{2}-\rho^{-8 \mu}\left(d x^{2}+d y^{2}\right),
$$

where

$$
\rho=\left(x^{2}+y^{2}\right)^{1 / 2} \text {. }
$$

As was first shown by Sokolov and Starobinskii [20], the corresponding energymomentum tensor has the form:

$$
\sqrt{g} T_{a b} \equiv \rho^{8 \mu} T_{a b}=\mu \delta^{(2)}(x, y)\left(\nabla_{a} t \nabla_{b} t-\nabla_{a} z \nabla_{a} z\right)
$$

and thus the constant $\mu$ is simply the mass per unit length of the line singularity representing the string, which can also be seen to have a longitudinal tension of strength $\mu c^{2}$.

Of course, to fully describe a realistic string it is necessary to supplement the line element (1.1) or (1.2) with an appropriate interior solution (see, for example, [16]). However, the thickness of a GUT string is likely to be of the order of $10^{-30} \mathrm{~cm} \mathrm{[28]}$ and so for all practical purposes the bare exterior solution is probably adequate.

A popular misconception that should at this point be refuted is that the energymomentum tensor of the bare straight string metric is not well defined as a distribution. This misleading claim stems from the work of Geroch and Traschen [9], who in attempt 
to place the theory of singular 2-surfaces on a more rigorous basis introduced a class of "regular metrics", which are characterised by the fact that $g_{a b}$ and its inverse are locally bounded, and the (generalised) first derivative of $g_{a b}$ is locally square integrable. A regular metric will always give rise to an energy-momentum tensor that is not worse than distributional, but a metric need not to be regular for $T_{a b}$ to be well behaved.

In fact a straight-string metric in its isotropic form (1.2) is an example of a metric which is not regular but nonetheless has an acceptable distributional energymomentum density (1.4). That is so follows immediately from the Gauss-Bonnet theorem, which states that the angle deficit $\Delta \theta$ is equal to the integral of the Gaussian 2-curvature $K$ over any surface of constant $z$ and $t$. The density $\sqrt{|g|} K$ must therefore be a distribution, and it is easily seen from the symmetries of the metric that $T_{t t}=-T_{z z}=-K /(8 \pi G)$ and all other components of $T_{a b}$ are zero. The physical components of the Ricci and Riemann tensors constructed from $R_{x x}, R_{y y}$ and $R_{x y x y}$ are also well-behaved distributions, and are the only non-zero curvature components. All these features of the metric are implicit in the work of Sokolov and Starobinskii [20], but have been rederived using more sophisticated methods by a number of authors, including in particular Clarke, Vickers and Wilson [7].

A number of extensions of the basic straight-string metric have been published since 1985. These include metrics that describe a straight string with non-zero cosmological constant [23], a string passing through a black hole [1], $N$ straight parallel strings [15] and two straight non-parallel moving strings [11], as well as a series of solutions that use inverse scattering methods (among others) to model the interaction of cylindrical gravitational waves with a single straight string $[4,5,8,19,29,30]$. There is also a trio of solutions due to Stein-Schabes whose interpretation is uncertain [22].

Other modifications of the straight-string metric that I will not consider in this paper include spinning strings [17], superconducting strings [18] and the so-called "cosmic rings" [2], which are line singularities with a more general energy-momentum tensor than (1.4).

One generalisation of the straight-string metric that does have important ramifications for cosmic-string theory is the "travelling wave" solution of Garfinkle [7], which was prefigured by a weak-field result due to Vachaspati [26]. In its simplest form, the line element reads:

$$
d s^{2}=(1+F) d t^{2}-(1-F) d z^{2}-2 F d t d z-\rho^{-8 \mu}\left(d x^{2}+d y^{2}\right),
$$

where $\rho$ is as given in (1.3) and

$$
F=2 x A^{\prime \prime}(t-z)+2 y B^{\prime \prime}(t-z),
$$

with $A$ and $B$ arbitrary twice-differentiable functions of $t-z$. [Strictly speaking, these equations describe only one of the two possible modes that can appear in travelling 
wave solutions. The second mode can be found by replacing $t-z$ with $t+z$ everywhere, and reversing the sign of the $d z d t$ cross-term.]

A physical interpretation of the travelling wave solutions is suggested by making use of transformation

$$
\begin{gathered}
x=\bar{x}-A, \quad y=\bar{y}-B \\
t=\bar{t}-\bar{x} A^{\prime}-\bar{y} B^{\prime}+\frac{1}{2} \int_{0}^{\bar{i}-\bar{z}}\left[A^{\prime 2}(u)+B^{\prime 2}(u)\right] d u
\end{gathered}
$$

and

$$
z=\bar{z}-\bar{x} A^{\prime}-\bar{y} B^{\prime}+\frac{1}{2} \int_{0}^{\bar{t}-\bar{z}}\left[A^{\prime 2}(u)+B^{\prime 2}(u)\right] d u
$$

to rewrite the line element in the form:

$$
\begin{aligned}
& d s^{2}=d \bar{t}^{2}-d \bar{z}^{2}+2\left(\rho^{-8 \mu}-1\right)\left(A^{\prime} d \bar{x}+B^{\prime} d \bar{y}\right)(d \bar{t}-d \bar{z}) \\
& -\left(\rho^{-8 \mu}-1\right)\left(A^{\prime 2}+B^{\prime 2}\right)(d \bar{t}-d \bar{z})^{2}-\rho^{-8 \mu}\left(d \bar{x}^{2}+d \bar{y}^{2}\right) \text {, }
\end{aligned}
$$

where now $\rho=\left[(\bar{x}-A)^{2}+(\bar{y}-B)^{2}\right]^{1 / 2}$. [Note that $A$ and $B$ are interchangeably functions of $t-z$ or $\bar{t}-\bar{z}$, as this is an invariant of the transformation.]

The functional form of $\rho$ in (1.10) suggests that the travelling wave solution should be interpreted as the metric of a wave of arbitrary shape $\bar{x}=A(\bar{t}-\bar{z}), \bar{y}=B(\bar{t}-\bar{z})$ propagating up the string. This interpretation is reinforced by the fact that the solution (1.5) is a special case of a more general family of solutions with the same line element, but with

$$
F=\sum_{1} C_{i}^{\prime \prime}(z-t) q_{i}(x, y)
$$

where the $C_{i} \mathrm{~s}$ are arbitrary and the $q_{i}$ s are any harmonic functions [6]. Solutions of this type describe plane-fronted (pp) waves superposed on the bare straight-string metric (1.2) and bear a close resemblance to the standard pp waves generated by Minkowski spacetime. The crucial difference, however, is that for Minkowski pp waves the lowest-order (linear) harmonic is diffeomorphic to flat space (this can be seen by letting $\mu \rightarrow 0$ in (1.10)), whereas the string travelling wave solutions are not flat. This leads Frolov and Garfinkle [6] to conclude that travelling waves are intrinsic to the string itself: that in fact "the traveling-wave solution [is] the gravitational field of the moving string"

However, there are reasons for believing that Garfinkle's travelling wave solutions describe a special type of gravitational wave phenomenon peculiar to cosmic strings 
(whether static or moving) rather than a generic gravitational response to the presence of a moving string. In the first place, the line element (1.5) is stationary and nondissipative, in contrast to the well-grounded physical expectation that a moving string should radiate gravitational energy [28]. (It is in any case clear that the travelling wave metric cannot be used to describe an arbitrary string configuration, as the two modes cannot be linearly superposed.)

At a more geometric level, it can be shown that the world sheet of any travelling wave solution is intrinsically flat. In general, a moving string will be characterised by a non-flat world sheet, a feature which leads to gravitational dissipation and even the possibility of gravitational collapse. In order to demonstrate this, and also to place the study of the near field of a generic cosmic string on a firm mathematical foundation, it is necessary first of all to construct a co-ordinate system adapted to the geometry of the string's world sheet.

\section{A 3+1 split of the cosmic string metric}

The trajectory of an idealised (that is, infinitesimally thin) cosmic string traces out a 2-dimensional surface, or world sheet, in spacetime. Naively, the most natural way to construct spacetime co-ordinates that reflect the geometry of the world sheet would be to foliate spacetime with spacelike surfaces generated by geodesics that intersect the world sheet orthogonally ("normal surfaces"), erect a system of Gaussian polar co-ordinates on each normal surface and choose the two remaining co-ordinates to be constant on the normal surfaces.

This procedure is described in some detail in [25], but it remains a purely formal prescription unless supplemented by a much clearer definition of the concept of normal surface. The problem, as can be seen from the bare straight-string metric (1.2), is that the world sheet is a singular surface with no well-defined tangent space. It is therefore meaningless to claim that a particular geodesic intersects the world sheet "orthogonally".

Nonetheless, in the case of the straight-string metric (1.1) or (1.2) it is clear that the desired normal surfaces are just surfaces of constant $z$ and $t$. What distinguishes these surfaces is the fact that the angle deficit $\Delta \theta$ assumes a critical value on each of them. This is best appreciated by referring to the cylindrical form of the metric in (1.1). A general spacelike geodesic radiating from a fixed point $\mathbf{p}=\left(t_{0}, z_{0}\right)$ on the world sheet has the form

$$
\begin{array}{ll}
t=t_{0}+\varepsilon \sinh \chi, & z=z_{0}+\varepsilon \cosh \chi \sin \psi, \\
r=\varepsilon \cosh \chi \cos \psi, & \psi=\text { constant },
\end{array}
$$

where $\chi \in \Re$ and $\psi \in(-\pi / 2, \pi / 2)$ are fixed parameters, and $\varepsilon$ is the proper distance 
from the world sheet. If $\mathbf{T}$ is a spacelike surface generated by a family of such geodesics then $\chi$ and $\psi$ are typically functions of $\phi$ on $\mathbf{T}$.

The induced line element on the surface $\mathbf{T}$ is easily shown to be:

$$
d s^{2}=-d \varepsilon^{2}-\left(\alpha^{2} \cosh ^{2} \chi \cos ^{2} \psi+\cosh ^{2} \chi \dot{\psi}^{2}-\dot{\chi}^{2}\right) \varepsilon^{2} d \phi^{2},
$$

where an overdot denotes the derivative with respect to $\phi$. The corresponding angle deficit is:

$$
\Delta \theta=2 \pi-\int_{0}^{2 \pi} \sqrt{\alpha^{2} \cosh ^{2} \chi \cos ^{2} \psi+\cosh ^{2} \chi \dot{\psi}^{2}-\dot{\chi}^{2}} d \phi .
$$

If $\chi$ and $\psi$ are constant, the angle deficit is

$$
\Delta \theta=2 \pi(1-\alpha \cosh \chi \cos \psi)
$$

and has the unique critical value $2 \pi(1-\alpha) \equiv 8 \pi \mu$ when $\chi=\psi=0$ (that is when $T$ is a surface of constant $t$ and $z$ ).

In the more general case where $\chi$ and $\psi$ vary with $\phi$, the question of which surfaces give a critical value of $\Delta \theta$ reduces to a standard variational problem with Lagrangian

$$
L=\int_{0}^{2 \pi} \sqrt{\alpha^{2} \cosh ^{2} \chi \cos ^{2} \psi+\cosh ^{2} \chi \dot{\psi}^{2}-\dot{\chi}^{2}} d \phi
$$

provided that $\chi$ and $\psi$ are differentiable functions of $\phi$. The corresponding EulerLagrange equations have two immediate first integrals: the energy functional

$$
E \equiv \dot{\chi} \frac{\partial L}{\partial \dot{\chi}}+\dot{\psi} \frac{\partial L}{\partial \dot{\psi}}-L=-\alpha^{2} L^{-1} \cosh ^{2} \chi \cos ^{2} \psi
$$

and a second functional which can be found by rearranging and integrating the equation for $x$ :

$$
C=L^{-2} \cosh ^{2} \chi \dot{\chi}+\cosh ^{2} \chi .
$$

Solving for $\chi$ and $\psi$ gives:

$$
\dot{\chi}^{2}=\left(C-\cosh ^{2} \chi\right) E^{-2} \alpha^{4} \cosh ^{2} \chi \cos ^{4} \psi
$$

and

$$
\dot{\psi}^{2}=C E^{-2} \alpha^{4} \cos ^{2} \psi\left(\cos ^{2} \psi-\cos ^{2} \psi_{0}\right),
$$


where $\cos \psi_{0} \equiv|E| /(\sqrt{C} \alpha)$. If $\psi$ is not constant, it must oscillate from $-\psi_{0}$ to $\psi_{0}$ and back a total of $n$ times (where $n$ is some natural number) as $\phi$ varies around the surface $T$. Hence from equation (2.9),

$$
2 \pi=\int_{0}^{2 \pi} d \phi=4 n \alpha^{-1} \int_{0}^{\psi_{0}} \frac{\cos \psi_{0}}{\cos \psi\left(\cos ^{2} \psi-\cos ^{2} \psi_{0}\right)} d \psi=2 \pi n \alpha^{-1} .
$$

Since $\alpha<1$ this equation can never be satisfied, and so $\psi$ must be constant [and in fact must be either $\psi-0$ or $-\psi_{0}$, as the other potential root of $(2.9), \cos \psi=0$, collapses $\mathbf{T}$ into a line on the world sheet] on any surface with critical angle deficit.

If $\chi$ is not constant on such a surface, and $\cosh \chi_{0} \equiv C$, then $\chi$ must oscillate from $-\chi_{0}$ to $\chi_{0}$ and back again a total of $n$ times, and equation (2.8) integrates to give:

$$
2 \pi=4 n \alpha^{-1} \sec \psi_{0} \int_{0}^{\psi_{0}} \frac{\cosh \chi_{0}}{\cosh \chi\left(\cosh ^{2} \chi-\cosh ^{2} \chi_{0}\right)} d \chi=2 \pi n \alpha^{-1} \sec \psi_{0} .
$$

Again, this equation can never be satisfied, $\chi$ must also be a constant, and the problem reduces to finding the critical values of equation (2.4). Hence, the only surfaces $\mathbf{T}$ on which $\Delta \theta$ assumes a critical value are the surfaces of constant $z$ and $t$, as claimed.

This result suggests the following prescription for constructing a suitable set of co-ordinates in some neighbourhood of the world sheet of a non-straight string. Any 2 -surface $\mathbf{T}$ generated by spacelike geodesics radiating from a single point $\mathbf{p}$ on the world sheet $\mathbf{S}$ has a conical singularity at $\mathbf{p}$. The strength of the singularity is measured by its angle deficit $\Delta \theta$, which is defined as follows. Let $\{\gamma(\varepsilon)\}$ be any family of closed spacelike curves on $\mathbf{T}$ that enclose $\mathbf{p}$ with winding number 1 and have the property that $\lim _{\varepsilon \rightarrow 0} \gamma(\varepsilon)=\mathbf{p}$. Then, by virtue of the Gauss-Bonnet theorem,

$$
\Delta \theta=2 \pi-\lim _{\varepsilon \rightarrow 0} \int_{r(\varepsilon)} \kappa_{g} d s,
$$

where $\kappa_{g}$ is the geodesic curvature of $\gamma(\varepsilon)$, and $s$ measures proper distance along $\gamma(\varepsilon)$. The normal surface $\mathbf{N}_{\mathbf{p}}$ is defined to be the unique conical 2-surface $\mathbf{T}$ generated by spacelike geodesics radiating from $\mathbf{p}$ whose angle deficit $\Delta \theta$ has the critical value $8 \pi \mu$. The existence of a normal surface through each point on the world sheet will be regarded as a defining feature of a (non-spinning) string metric.

To choose a set of co-ordinates tailored to the geometry of the normal surfaces, it is necessary first of all to select a pair of co-ordinates $\zeta^{A}=\left(\zeta^{0}, \zeta^{1}\right)$ on the world sheet S. The choice of $\zeta^{A}$ is for the moment arbitrary, but will later be restricted by certain gauge conditions. If the co-ordinate $\eta$ is chosen to be any parameter running from 0 to $2 \pi$ that smoothly parameterises the geodesics generating $\mathbf{N}_{\mathbf{p}}$ for each $\mathbf{p}$ on $\mathbf{S}$, and the co-ordinate $r$ is chosen to be the proper distance from $\mathbf{p}$ along each of the geodesics, then it is possible to assign the unique co-ordinates $x^{a}=\left(\zeta^{A}(\mathbf{p}), r, \eta\right)$ to every point 
on $\mathbf{N}_{\mathbf{p}}$ in some neighbourhood of $\mathbf{S}$. In fact, these co-ordinates are well-defined out to the local radius of curvature of the world sheet, where neighbouring normal surfaces first begin to cross.

Since lines of constant $\zeta^{A}$ and $\eta$ are spacelike geodesics, and $r$ measures their proper length, the metric components in the tailored co-ordinates satisfy

$$
g_{r r}=-1 \text { and } g_{a r \cdot r}=0 .
$$

Furthermore, since each normal surface is by assumption spacelike, the discriminant $\Delta=g_{r r} g_{\eta \eta}-\left(g_{r \eta}\right)^{2}$ is positive everywhere except possibly on the world sheet. If each point $\mathbf{p}$ on the world sheet has the exterior geometry of a point rather than a ring (so that the circumference of any circle $r=\varepsilon$ goes to zero as $\varepsilon \rightarrow 0$ ) the $\lim _{r \rightarrow 0} g_{\eta \eta}=0$. It therefore follows that, since $g_{r r} g_{\eta \eta}$ tends to zero as $r$ goes to zero, $g_{r \eta}$ also tends to zero at the world sheet. in view of (2.13) this means that $g_{r \eta}=0$ everywhere. ${ }^{2}$

Combining this with the equation for the angle deficit on $\mathbf{N}_{\mathbf{p}}$ gives:

$$
8 \pi \mu=2 \pi-\lim _{r \rightarrow 0} \int_{0}^{2 \pi} \frac{\partial}{\partial r}\left(-g_{\eta \eta}\right)^{1 / 2} d \eta
$$

and so the metric component $g_{\eta \eta}$ has the near-field expansion

$$
\left(-g_{\eta \eta}\right)^{1 / 2} \sim h\left(\zeta^{A}, \eta\right) r
$$

where $h$ is some function with the property that

$$
\int_{0}^{2 \pi} h\left(\zeta^{A}, r\right) d \eta=2 \pi(1-4 \mu)
$$

If $\eta$ is now replaced with a new co-ordinate $\phi\left(\zeta^{A}, \eta\right)$ defined through the equation

$$
\frac{\partial \phi}{\partial \eta}=(1-4 \mu)^{-1} h\left(\zeta^{A}, \eta\right) \quad \text { with } \quad \phi\left(\zeta^{A}, 0\right)=0
$$

then $\phi$ runs from 0 to $2 \pi$ on each normal surface, and for small values of $r$,

$$
g_{\phi \phi} \sim-(1-4 \mu)^{2} r^{2} .
$$

Also, the metric component $g_{r \phi}$ (like $g_{r \eta}$ ) is everywhere zero.

\footnotetext{
${ }^{2}$ It should be noted that this construction does not apply in the case of a bare spinning string [17]. There, the world sheet is characterised by a ring singularity rather than a simple conical singularity, a fact intimately related to the fact that bare spinning strings support closed timelike curves and are unlikely to be physical [21].
} 
The first variation of the angle deficit in the neighbourhood of each normal surface $\mathbf{N}_{\mathbf{p}}$ can be calculated by examining spacelike surfaces with the parametric representation $\left\{r=\varepsilon, \zeta^{A}=\varepsilon v^{A}(\phi)\right\}$, where the $v^{A}$ are arbitrary differentiable functions with period $2 \pi$. To linear order in $v^{A}$ the line element induced on such a perturbation surface is:

$$
d s^{2}=\left(-1+2 g_{A r} v^{A}\right) d \varepsilon^{2}+2\left(\varepsilon g_{A r} v_{r_{\phi}}^{A}+g_{A \phi} v^{A}\right) d \varepsilon d \phi+\left(g_{\phi \phi}+2 \varepsilon g_{A \phi} v_{r_{\phi}}^{A}\right) d \phi^{2}
$$

and so in view of (2.12) the corresponding angle deficit is:

$$
\Delta \theta=2 \pi(1-\alpha)-\lim _{r \rightarrow 0} \int_{0}^{2 \pi}\left(\alpha^{-1} g_{A \phi^{\prime} \cdot \phi}+\alpha g_{A r}\right) v^{A} d \phi
$$

Hence, the requirement that $\Delta \theta$ be critical on each normal surface is equivalent to the co-ordinate condition:

$$
\lim _{r \rightarrow 0}\left[g_{A \phi \cdot r \phi}+(1-4 \mu)^{2} g_{A r}\right]=0 .
$$

Furthermore, if the metric components $g_{A B}$ are assumed to have well-defined limits on the world sheet, then for small values of $r$ the Ricci components $R_{A B}$ have the leading order form

$$
R_{A B} \sim-\frac{1}{4} g^{r r} g^{\phi \phi}\left(g_{A r^{\prime} \phi}-g_{A \phi}\right)\left(g_{B r^{\prime} \phi}-g_{B \phi} \cdot r\right) .
$$

For a cosmic string in vacuum, therefore,

$$
\lim _{r \rightarrow 0}\left(g_{A r \cdot \phi}-g_{A \phi \cdot r}\right)=0 .
$$

Recall from (2.13) that the metric component $g_{A r}$ is independent of $r$. If it is assumed that $g_{A \phi}$ is sufficiently well-behaved that the limit and the derivative with respect to $\phi$ can be interchanged in (2.21), then (2.21) and (2.23) can be combined to give the second-order equation:

$$
g_{A r \phi \phi}+(1-4 \mu)^{2} g_{A r}=0,
$$

which needs to be solved subject to the periodicity condition $g_{A r}=g_{A r}$. It is clear by inspection that unless $\mu=0$ (2.24) has no non-trivial periodic solution, and so $g_{\text {Ar }}=0$.

In summary, the line element of a generic cosmic string in vacuum in some neighbourhood of the world sheets can always be written in what I will henceforth refer to as the canonical form:

$$
d s^{2}=g_{A B} d \zeta^{A} d \zeta^{B}+2 g_{A \phi} d \zeta^{A} d \phi+g_{\phi \phi} d \phi^{2}-d r^{2},
$$


with $g_{\phi \phi} \sim-\alpha^{2} r^{2}$ for small values of $r$, and from (2.23)

$$
\lim _{r \rightarrow 0} g_{A \phi, r}=0 \text {. }
$$

It has been assumed in deriving (2.23), that the metric components $g_{A B}$ have welldefined limits on $S$, and so

$$
\gamma_{A B}=\lim _{r \rightarrow 0} g_{A B}
$$

is a function of the world-sheet co-ordinates $\zeta^{A}$ alone. $^{3}$

The canonical form (2.25) of the near-field metric is of course not unique, as there is considerable freedom in the choice of the co-ordinates $\zeta^{A}$. However, it is always possible to choose these co-ordinates so that the induced 2-metric is conformally flat:

$$
\gamma_{A B}=\kappa \eta_{A B},
$$

where $\eta_{A B}=\operatorname{diag}(1,-1)$ and $\kappa$ some function of $\zeta^{A}$. In terms of $\kappa$, the intrinsic curvature $R^{(2)}$ of the world sheet is

$$
R^{(2)}=\kappa^{-1} \eta^{A B}(\ln \kappa) \cdot_{A B} .
$$

A less obvious source of gauge freedom in (2.25) is the fact that the zero point of the angular co-ordinate $\phi$ on each of the normal surfaces $\mathbf{N}_{\mathbf{p}}$ is arbitrary, and so the general form of the line element is preserved under point rotations of the form:

$$
\phi \rightarrow \phi^{\prime}=\phi+\psi\left(\zeta^{A}\right)
$$

\section{Travelling waves versus curvature waves}

A series solution for the generic cosmic-string metric can be generated by substituting the canonical line element (2.25) into the vacuum Einstein equations. This will be done shortly, but it is instructive to first cast two of the known exact solutions into canonical form.

The generalised travelling-wave solutions described by (1.5) and (1.10) are characterised in canonical co-ordinates by the following metric components:

$$
g_{\phi \phi}=-\alpha^{2} r^{2}, \quad g_{A \phi}=0 \quad \text { and } \quad g_{A B}=\eta_{A B}+\left[\begin{array}{cc}
F & -F \\
-F & F
\end{array}\right]_{A B},
$$

\footnotetext{
${ }^{3}$ It is evident from (2.25) that the cosmic-string metrics, as defined here, form a subclass of the class of "line singularities" introduced by Israel in 1977 [13]. In particular, the normal surfaces are generated by spacelike geodesics that extremise the proper distance from the world sheet. However, the cosmic-string metrics do not in general belong to Israel's class of "simple line sources", as they fail to satisfy the condition Israel calls "parity invariance", which is equivalent to requiring the world sheet to be twist-free (see below).
} 
where

$$
F=2 \sum_{n=1}^{\infty} r^{n / \alpha}\left[\cos (n \phi) A_{n}^{\prime \prime}(t-z)+\sin (n \phi) B_{n}^{\prime \prime}(t-z)\right]
$$

for any choice of functions $A_{n}$ and $B_{n}$. The most important features of the solutions to note are that the deviation from the bare straight-string metric (1.1) proceeds as a power series in $r^{1 / \alpha}$ (where $1 / \alpha>1$ ), and that $\kappa=1$ and so the world sheet is intrinsically flat.

By way of contrast, the simplest known solution containing curvature waves is the Aryal-Ford-Vilenkin metric [1], which describes a cosmic string passing through a black hole. The line element is:

$$
d s^{2}=\left(1-\frac{2 m}{\rho}\right) d t^{2}-\left(1-\frac{2 m}{\rho}\right)^{-1} d \rho^{2}-\rho^{2} d \chi^{2}-\alpha^{2} \rho^{2} \sin ^{2} \chi d \phi^{2}
$$

and can be constructed by removing a wedge of angular extent $\Delta \theta=2 \pi(1-\alpha)$ from along any axis of the Schwarzschild metric. The metric (3.3) can be rewritten in canonical form by replacing $\rho$ and $\chi$ with two new co-ordinates $r$ and $z$ defined implicitly by the equations:

$$
r= \pm \int_{z}^{\rho}\left[\left(1-\frac{2 m}{u}\right)\left(1-\frac{z^{2}}{u^{2}}\right)\right]^{-1 / 2} d u
$$

and

$$
\chi= \pm z \int_{z}^{\rho}\left[\left(1-\frac{2 m}{u}\right)\left(1-\frac{z^{2}}{u^{2}}\right)\right]^{-1 / 2} u^{-2} d u
$$

where the positive branch applies outside the event horizon $(\rho>2 m)$ and the negative branch inside the event horizon $(\rho<2 m)$. The canonical line element is too complicated to be reproduced in full, but for small values of $r$ it has the form:

$$
\begin{aligned}
d s^{2} \sim & \left(1-\frac{2 m}{z}\right)\left(1+\frac{m}{z^{3}} r^{2}\right) d t^{2}-\left(1-\frac{2 m}{z}\right)^{-1}\left(1+\frac{m}{z^{3}} r^{2}\right) d z^{2} \\
& -\alpha^{2} r^{2}\left(1-\frac{2 m}{3 z^{3}} r^{2}\right) d \phi^{2}-d r^{2}
\end{aligned}
$$

In fact, the asymptotic form of the line element can be made even more compact by transforming to Kruskal-Szekeres co-ordinates, in terms of which the 2-metric induced on the world sheet is conformally flat:

$$
\begin{aligned}
d s^{2} \sim & 16 m^{2} z^{-1} e^{-z / 2 m}\left(1+\frac{m}{z^{3}} r^{2}\right)\left(d \tau^{2}-d \sigma^{2}\right) \\
& -\alpha^{2} r^{2}\left(1-\frac{2 m}{3 z^{3}} r^{2}\right) d \phi^{2}-d r^{2}
\end{aligned}
$$


where $z$ should now be regarded simply as a parameter which is given by the world sheet co-ordinates $\tau$ and $\sigma$ through the relation:

$$
\tau^{2}-\sigma^{2}=(2 m-z) e^{z / 2 m} .
$$

Two features of the Aryal-Ford-Vilenkin metric highlighted by the canonical line element (3.7) are the fact that the near-field expansion proceeds in powers of $r^{2}$ rather than powers of $r^{1 / \alpha}$, and the fact that the world sheet is not intrinsically flat, as the conformal factor

$$
\kappa=16 m^{2} z^{-1} e^{-z / 2 m}
$$

generates a curvature invariant of the form:

$$
R^{(2)}=\frac{4 m}{z^{3}}
$$

The canonical form (2.25) is thus able to distinguish between two fundamentally different types of gravitational field in the neighbourhood of a generic cosmic string. Moreover, it is clear by comparison of (3.7) and (3.10) that the extrinsic curvature $R^{(2)}$ effectively determines the scale of the second-order expansion terms in $g_{A B}$ and $g_{\phi \phi}$. As will be seen in Section 4, the existence of an intimate relationship between the world-sheet curvature and the near-field expansion is a general feature of all metrics containing curvature waves.

\section{A general near-field expansion of the metric}

A series solution to the vacuum Einstein equations for the canonical line element (2.25) can be developed by expanding the metric components in undetermined powers of the geodesic distance $r$ :

$$
\begin{gathered}
g_{A B}=\kappa\left(\eta_{A B}+\sum_{n=1}^{\infty} P_{A B}^{(n)} r^{j_{n}}\right), \\
g_{A \phi}=\sum_{i=n}^{\infty} Q_{A}^{(n)} r^{k_{n}}
\end{gathered}
$$

and

$$
g_{\phi \phi}=-\alpha^{2} r^{2}\left(1-\sum_{n=1}^{\infty} \beta^{(n)} r^{l_{n}}\right)
$$


where the sequences of exponents $\left\{j_{n}\right\},\left\{k_{n}\right\}$ and $\left\{l_{n}\right\}$ are positive and ascending, but for the moment otherwise unrestricted (save that $k_{1}$ is strictly greater than 1 as required by (2.26)). To leading order in $r$, the corresponding components of the Ricci tensor are:

$$
\begin{aligned}
& R_{A B} \sim \frac{1}{2} \kappa R^{(2)} \eta_{A B}-\frac{1}{2} \kappa \alpha^{-2}\left(P_{A B^{\prime} \phi \phi}^{(1)}+\alpha^{2} j_{1}^{2} P_{A B}^{(1)}\right) r^{j_{1}-2} \\
& -\frac{1}{2} \alpha^{-2} k_{1}^{2} Q_{A}^{(1)} Q_{B}^{(1)} r^{2 k_{1}-4}+\alpha^{-2}\left[Q_{\left(A^{\prime} B\right) \phi}^{(1)}-\kappa^{-1} \kappa^{\prime} \cdot(A) Q_{B)^{\prime} \phi}^{(1)}\right. \\
& \left.+\frac{1}{2} \kappa^{-1} \eta_{A B} \eta^{C D} \kappa^{\prime} C Q_{D \cdot \phi}^{(1)}\right] r^{k_{1}-2} \text {, } \\
& R_{A \phi} \sim \frac{k_{1}\left(2-k_{1}\right)}{2} Q_{A}^{(1)} r^{k_{1}-2}+\frac{1}{2} \eta^{C D}\left(P_{C D^{\circ} A \phi}^{(1)}-P_{A C \cdot D \phi}^{(1)}\right) r^{j_{1}} \\
& +\frac{1}{2} \eta^{C D}\left(\frac{1}{2} \kappa^{-1} \kappa \cdot A P P_{C D \cdot \phi}^{(1)}-\kappa^{-1} \kappa \cdot C P_{A D \cdot \phi}^{(1)}\right) r^{j /}, \\
& R_{A r} \sim \frac{k_{1}}{2} \alpha^{-2} Q_{A}^{(1)} r^{k_{1}-3}-\frac{1}{2}\left(l_{1}+1\right) \beta_{{ }_{A}}^{(1)} r^{l_{1}-1} \\
& +\frac{j_{1}}{2} \eta^{C D}\left(P_{C D, A}^{(1)}-P_{A C \cdot D}^{(1)}+\frac{1}{2} \kappa^{-1} \kappa^{{ }^{\prime} \cdot A} P_{C D}^{(1)}-\kappa^{-1} \kappa^{\prime} C P_{A D}^{(1)}\right) r^{j_{1}-1} \\
& R_{\phi \phi} \sim-\kappa^{-1} \eta^{C D} Q_{C \cdot D \phi}^{(1)} r^{k_{1}}+\left(\frac{1}{2} k_{l}^{2}-k_{1}+1\right) \kappa^{-1} \eta^{C D} Q_{C}^{(1)} Q_{D}^{(1)} r^{2\left(k_{1}-1\right)} \\
& +\frac{1}{2} \eta^{C D}\left(P_{C D, \phi \phi}^{(1)}+\alpha^{2} j_{1} P_{C D}^{(1)}\right) r^{j_{1}}-\frac{1}{2} \alpha^{2} l_{1}\left(l_{1}+1\right) \beta^{(1)} r^{l_{1}}, \\
& R_{r \phi} \sim \frac{j_{1}-1}{2} \eta^{C D} P_{C D}^{(1)}{ }_{\phi} r^{j_{1}-1}+\frac{2-k_{1}}{2} \kappa^{-1} \eta^{C D} Q_{C \cdot D}^{(1)} r^{k_{1}-1} \\
& +\frac{k_{1}}{2} \alpha^{-2} \kappa^{-1} \eta^{C D} Q_{C}^{(1)} Q_{D^{\prime} \phi}^{(1)} r^{2 k_{1}-3}
\end{aligned}
$$

and

$$
\begin{aligned}
R_{r r} \sim & \frac{j_{1}\left(j_{1}-1\right)}{2} \eta^{C D} P_{C D}^{(1)} r^{j_{1}-2}-\frac{l_{1}\left(l_{1}+1\right)}{2} \beta^{(1)} r^{l_{1}-2} \\
& +\alpha^{-2}\left(\frac{3}{2} k_{1}^{2}-3 k_{1}+1\right) \kappa^{-1} \eta^{C D} Q_{C}^{(1)} Q_{D}^{(1)} r^{2 k_{1}-4},
\end{aligned}
$$

where the subscripted brackets in (4.4) denote symmetrisation.

For a cosmic string isolated in vacuum, all components of the Ricci tensor should of course be set to zero. From (4.5), this is possible only if $k_{1} \geq 2$; while from (4.4) it is either the case that $j_{1} \geq 2$ or that

$$
P_{A B \cdot \phi \phi}^{(1)}+\alpha^{2} j_{1}^{2} P_{A B}^{(1)}=0
$$


which yields a non-trivial solution only if $j_{1}=n / \alpha$ for some integer $n$. Very broadly, these two possibilities correspond to the existence of lowest-order travelling waves $\left(j_{1}=1 / \alpha\right)$ and the existence of curvature waves in the absence of lowest-order travelling waves $\left(j_{1}=2\right.$ ). In what follows I will assume that $1 / \alpha<2$ (which corresponds to $\mu<1 / 8)$.

In the case $j_{1}=1 / \alpha,(4.10)$ can be solved to give:

$$
P_{A B}^{(1)}=L_{A B} \cos \phi+M_{A B} \sin \phi
$$

where the coefficient matrices $L_{A B}$ and $M_{A B}$ are functions of the world-sheet coordinates alone. In view of (4.8), the coefficient matrices are traceless:

$$
\eta^{C D} L_{C D}=\eta^{C D} M_{C D}=0
$$

while (4.7) or (4.9) requires that $l_{1}>j_{1}$. [In fact, a higher-order expansion of the Ricci components indicates that $l_{1} \geq 2$.] The only other non-trivial constraint at this order stems from (4.6), which requires that either $k_{1} \geq j_{1}+2>3$ or

$$
Q_{A}^{(1)}=0 .
$$

Of course, the lowest-order travelling wave solutions of the form (3.1) satisfy all these constraints, as well as other restrictions generated by higher-order terms in the Einstein equations. There is no obstruction to curvature waves (with $j_{2}=k_{1}=l_{1}=2$ ) appearing on top of the lowest-order travelling waves, but one of the salient features of the lowest-order travelling waves is that they are independent of the conformal factor $\kappa$ and thus of the intrinsic curvature of the world-sheet.

In the case $j_{1}=2$, assuming the minimum possibilities $k_{1}=2$ and $l_{1}=2$, (4.6) leads directly to (4.13), while (4.4) and (4.7-4.9) can be combined to give the constraints:

$$
P_{A B, \phi}^{(1)}=0, \quad \beta^{(1)}=\frac{1}{6} R^{(2)} \quad \text { and } \quad P_{A B}^{(1)}+\alpha^{-2} \kappa^{-2} Q_{A}^{(1)} Q_{B}^{(1)}=\frac{1}{4} R^{(2)} \eta_{A B} .
$$

What is interesting to note about (4.13) and (4.14) is that the leading-order curvaturewave expansion terms are independent of the angular variable $\phi$, but depend critically on the intrinsic curvature $R^{(2)}$. In fact if the world sheet is flat then the only possible source of second-order contributions is a non-zero metric function $Q_{A}^{(1)}$ which, as will be seen shortly, is closely connected with the nature of the embedding of the string in the spacetime. Furthermore, the Aryal-Ford-Vilenkin metric (3.6) gives a surprisingly general example of the near-field expansion of a curvature-wave solution. The only restrictive feature of (3.6) is that $Q_{A}^{(1)}=0$, as the string involved is geometrically straight; otherwise the relationship between the first-order expansion terms and the 
world sheet curvature in the general case (4.13) and (4.14) can effectively be read off from (3.7) and (3.10).

The geometric significance of a non-zero metric function $Q_{A}^{(1)}$ can be illustrated by rewriting the Minkowski metric in terms of a set of co-ordinates $\left(\zeta^{a}, r, \phi\right)$ tailored to the geometry of a family of surfaces passing orthogonally through a fictious timelike surface $\mathbf{S}^{\prime}=\left\{x^{a}=X^{a}\left(\zeta^{0}, \zeta^{1}\right)\right\}$ in the same way that the canonical metric (2.25) is tailored to the geometry of the normal surfaces. In these co-ordinates the Minkowski line element becomes:

$$
\begin{aligned}
d s^{2}= & \left(\gamma_{A B}+2 x^{i} K_{(i) A B}+x^{i} x^{j} \gamma^{C D} K_{(i) A C} K_{(j) B D}-r^{2} \omega_{A} \omega_{B}\right) d \zeta^{A} d \zeta^{B} \\
& +2 r^{2} \omega_{A} d \zeta^{A} d \phi-d r^{2}-r^{2} d \phi^{2},
\end{aligned}
$$

where $\gamma_{A B}$ is the 2-metric induced on $\mathbf{S}^{\prime}, x^{i}=[r \cos \phi, r \sin \phi]$ and $K_{(i) A B}$ and $\omega_{A}$ are the extrinsic curvature tensor and twist vector of the surface $S^{\prime}$, respectively. If $t_{A}^{a} \equiv X^{a}{ }_{A}$ are vectors tangent to $\mathbf{S}^{\prime}$ and $n_{(i)}^{a}$ are orthogonal unit normals on $\mathbf{S}^{\prime}$ in the directions of $\partial x^{a} / \partial x^{i}$ then

$$
K_{(i) A B}=t_{(A} \cdot n_{(i) \cdot B)} \quad \text { and } \quad \omega_{A}=n_{(2)} \cdot n_{(1) \cdot A} \cdot
$$

Comparison of the line element (4.15) with the near-field expansions (4.1) and (4.2) indicates that the metric function $Q_{A}^{(1)}$ plays the same role as the twist vector $\omega_{A}$. In particular, if $Q_{A}^{(1)}$ is a pure gradient it can always be transformed away by redefining $\phi$, just as $\omega_{A}$ can.

The interpretation of the metric function $P_{A B}^{(1)}$ is more complicated, however. If $\mathbf{T}$ is any surface of constant $r$ and $\phi$ in the canonical cosmic-string metric (2.25), then to leading order in $r$ and $\phi$ components of the extrinsic curvature tensor on $\mathbf{T}$ are

$$
K_{A B}^{(r)} \sim-\frac{j_{1}}{2} \kappa P_{A B} r^{j_{1}-1}
$$

and

$$
\begin{aligned}
& K_{A B}^{(\phi)} \sim \alpha^{-1}\left[Q_{(A, B)}^{(1)}-\kappa^{-1} \kappa^{\cdot} \cdot{ }_{A} Q_{B)}^{(1)}+\frac{1}{2} \kappa^{-1} \eta^{C D} \kappa^{\prime} \cdot C Q_{D}^{(1)} \eta_{A B}\right] r^{k_{1}-1} \\
& -\frac{1}{2} \alpha^{-1} P_{A B^{\prime} \phi}^{(1)} r^{j_{1}-1}
\end{aligned}
$$

where subscripted brackets again denote symmetrisation. In the case of pure curvature waves, the $\phi$ component of the extrinsic curvature tensor tends to zero linearly in $r$, and to leading order is a linear functional of the metric function $Q_{A}^{(1)}$. In the case of the lowest-order travelling waves $\left(j_{1}=1 / \alpha\right)$, on the other hand, both the $r$ and $\phi$ components of the extrinsic curvature tensor tend to zero as $r^{1 / \alpha-1}$, a fact that was first established in [25]. Thus the extrinsic curvature of the world sheet itself is strictly 
zero, but the extrinsic curvature of neighbouring surfaces of constant $r$ and $\phi$ are (to leading order in $r$ ) linear functionals of $P_{A B}^{(1)}$.

A similar small-distance expansion of the Riemann tensor gives only three terms whose physical components diverge near the world sheet:

$$
\begin{aligned}
R_{A r B r} & \sim \frac{1}{2} \alpha^{-1}\left(\alpha^{-1}-1\right) \kappa P_{A B} r^{1 / \alpha-2}, \\
R_{A r B \phi} & \sim \frac{1}{2}\left(\alpha^{-1}-1\right) \kappa P_{A B{ }_{\phi} r^{1 / \alpha-1}}
\end{aligned}
$$

and

$$
R_{A \phi B \phi} \sim \frac{1}{2}(\alpha-1) \kappa P_{A B} r^{1 / \alpha} .
$$

In fact, the physical components of all three of these terms diverge as

$$
\left(\alpha^{-1}-1\right) r^{1 / \alpha-2} \sim \mu r^{4 \mu-1}
$$

(for small $\mu$ ), which is again in agreement with the analysis performed by Unruh et al. [25]. As can be seen, the divergence in the Riemann tensor is due solely to the contribution of the travelling waves; and the "characteristic global length" $a$ discussed by Unruh et al. [25] in connection with the divergence of the Riemann tensor is contained implicitly in the harmonic coefficients $L_{A B}$ and $M_{A B}$ appearing in $P_{A B}^{(1)}$.

In physical components, the remaining components of $R_{a b c d}$ all tend to zero as $r^{1 / \alpha-1}$, except for $R_{r \phi r \phi}$ and $R_{A B C D}$, which tend to finite, non-zero limits on the world sheet. In addition, The Riemann component $R_{r \phi r \phi}$ contains a singular distributional term, which is identical in structure to the singularity in $R_{r \phi r \phi}$ in the bare string metric (1.1). This last result is again a consequence of the Gauss-Bonnet theorem when applied to each of the normal surfaces.

\section{Conclusions}

In this paper I have developed a canonical line element that makes it possible to standardise and compare the near-field metrics of cosmic strings in vacuum. To leading order in the geodesic distance $r$ from the string's world sheet, the canonical metric supports terms of order $r^{1 / \alpha}$, which can be identified with the travelling waves first discovered by Garfinkle [7], and terms of order $r^{2}$, which appear in a large number of previously-published string solutions but have not so far been distinguished as a specific gravitational feature of cosmic strings. I have decided to call them curvature waves. 
Apart from their analytic structure, travelling waves and curvature waves differ in that the former are completely decoupled from the intrinsic curvature of the world sheet, whereas the latter couple directly to the curvature. In fact, the second-order terms in the expansion of a curvature-wave metric depend linearly on the scalar curvature of the world sheet, and there is a possibility that strings will experience some form of gravitational collapse whenever they develop a cusp (an isolated point on the world sheet where the motion of the string is luminal and the curvature is infinite $[14,24])$.

\section{Acknowledgments}

I would like to thank Edith Cowan University for sponsoring my attendance at the First Australian Workshop on General Relativity and Gravitation.

\section{References}

[1] M. Aryal, L. H. Ford and A. Vilenkin, "Cosmic strings and black holes", Phys. Rev. D34 (1986) 2263-2266.

[2] B. Carter, "Mechanics of cosmic strings", Phys. Lett. B238 (1990) 166-171.

[3] C. J. S. Clarke, J. A. Vickers and J. P. Wilson, "Generalised functions and distributional curvature of cosmic strings", Class. Quant. Grav. 13 (1996) 2485-2498.

[4] A. Economou and D. Tsoubelis, "Interaction of cosmic strings with gravitational waves: A new class of exact solutions", Phys. Rev. Lett. D61 (1988) 2046-2049.

[5] A. Economou and D. Tsoubelis, "Rotating cosmic strings and gravitational soliton waves", Phys. Rev. D38 (1988) 498-505.

[6] V. P. Frolov and D. Garfinkle, "Interaction of cosmic strings with gravitational waves", Phys. Rev. D42 (1990) 3980-3982.

[7] D. Garfinkle, "Traveling waves in strongly gravitating cosmic string", Phys. Rev. D41 (1990) $1112-1115$.

[8] J. Garriga and E. Verdaguer, "Cosmic strings and Einstein-Rosen soliton waves", Phys. Rev. D36 (1987) 2250-2258.

[9] R. Geroch and J. Traschen, "Strings and other distributional sources in general relativity", Phys. Rev. D36 (1987) 1017-1031.

[10] J. R. Gott, "Gravitational lensing effects of vacuum strings: Exact solutions", Astrophys. J. 288 (1985) 422-427.

[11] J. R. Gott, "Closed timelike curves produces by pairs of moving cosmic strings: Exact solutions", Phys. Rev. Lett. 66 (1991) 1126-1129.

[12] W. Hiscock, "Exact gravitational field of a string", Phys. Rev. D31 (1985) 3288-3290.

[13] W. Israel, "Line sources in general relativity", Phys. Rev. D15 (1977) 935-941.

[14] T. W. B. Kibble and N. Turok, "Self-intersection of cosmic strings", Phys. Rev. Lett. B116 (1982) 141-143.

[15] P. Letelier, "Multiple cosmic strings", Class. Quant. Grav. 4 (1987) L75-L77.

[16] B. Linet, "The static metrics with cylindrical symmetry describing a model of cosmic strings", Gen. Rel. Grav. 17 (1985) 1109-1115. 
[17] P. O. Mazur, "Spinning cosmic strings and quantization of energy", Phys. Rev. Lett. 57 (1986) 929-932.

[18] J. Ostriker, C. Thompson and E. Witten, "Cosmological effects of superconducting strings", Phys. Lett. B180 (1986) 231-239.

[19] D. Papadopoulos and B. C. Xanthopoulos, "Tomimatsu-Sato solutions describe cosmic strings interacting with gravitational waves", Phys. rev. D41 (1990) 2512-2518.

[20] D. D. Sokolov and A. A. Starobinskii, "The structure of the curvature tensor at conical singularities", Sov. Phys. Dokl. 22 (1977) 312-314.

[21] H. H. Soleng, "A spinning string", Gen. Rel. Grav. 24 (1992) 111-117.

[22] J. A. Stein-Schabes, "Nonstatic vacuum strings: Exterior and interior solutions", Phys. Rev. D33 (1986) 3545-3548.

[23] Q. Tian, "Cosmic strings with cosmological constant", Phys. Rev. D33 (1986) 3549-3555.

[24] N. Turok, "Grand unified strings and galaxy formation", Nucl. Phys. B242 (1984) 520-541.

[25] W. G. Unruh, G. Hayward, W. Israel and D. McManus, "Cosmic-string loops are straight", Phys. Rev. Lett. 62 (1989) 2897-2900, Note that the variables $d$ and $r$ used in this paper correspond to my $r$ and $\rho=(\alpha r)^{1 / \alpha}$ respectively.

[26] T. Vachaspati, "Gravitational effects of cosmic strings", Nucl. Phys. B277 (1986) 593-604.

[27] A. Vilenkin, "Gravitational field of vacuum domain", Phys. Rev. D23 (1981) 852-857.

[28] A. Vilenkin, "Cosmic strings and domain walls", Phys. Rep. 121 (1985) 263-315.

[29] B. C. Xanthopoulos, "Cylindrical waves and cosmic strings of Petrov type D", Phys. Rev. D34 (1986) 3608-3616.

[30] B. C. Xanthopoulos, "A rotating cosmic string", Phys. Lett. B178 (1986) 163-166. 